\title{
Core-shell Gold Nanorod@Zirconium-based metal-organic framework composites as in situ size-selective Raman probes
}

\author{
Johannes W. M. Osterrieth ${ }^{1, \S}$, Demelza Wright ${ }^{2, \S}$, Hyunho Noh ${ }^{3}$, Chung-Wei Kung ${ }^{3}$, Diana Vulpe ${ }^{1}$, Aure- \\ lia $\mathrm{Li}^{1}$, Ji Eun Park ${ }^{3}$, Richard P. Van Duyne ${ }^{3}$, Peyman Z. Moghadam ${ }^{1,4}$, Jeremy J. Baumberg ${ }^{2, *}$ Omar K. \\ Farha ${ }^{3,5,6, *}$ and David Fairen-Jimenez ${ }^{1, *}$
}

\begin{abstract}
${ }^{1}$ Adsorption and Advanced Materials (AAM) Laboratory, Department of Chemical Engineering and Biotechnology, University of Cambridge, Cambridge CB3 OAS, UK. ${ }^{2}$ NanoPhotonics Centre, Cavendish Laboratory, Department of Physics, University of Cambridge, Cambridge CB3 OHE, UK. ${ }^{3}$ Department of Chemistry, Northwestern University, Evanston, Illinois 60208, United States. ${ }^{4}$ Department of Chemical and Biological Engineering, University of Sheffield, Mappin Street, Sheffield S1 3JD, UK ${ }^{5}$ Department of Chemical and Biological Engineering, Northwestern University, Evanston, IL, 30208, United States. ${ }^{6}$ Department of Chemistry, Faculty of Science, King Abdulaziz University, Jeddah 21589, Saudi Arabia
\end{abstract}

${ }^{\S}$ Equal contribution; *Corresponding authors: jjb12@cam.ac.uk; farha-ofc@northwestern.edu; df334@cam.ac.uk

\begin{abstract}
Nanoparticle encapsulation inside zirconium-based metal-organic frameworks (NP@MOF) is hard to control and the resulting materials often have non-uniform morphologies with NPs on the external surface of MOFs and NP aggregates inside the MOFs. In this work, we report the controlled encapsulation of gold nanorods (AuNRs) by a scu-topology Zr-MOF, via a room-temperature MOF assembly. This is achieved by functionalizing the AuNRs with polyethylene glycol (PEG) surface ligands, allowing them to retain colloidal stability in the precursor solution and to seed the MOF growth. Using this approach, we achieve core-shell yields exceeding 99\%, tuning the MOF particle size via the solution concentration of AuNRs. The functionality of AuNR@MOFs is demonstrated by using the AuNRs as embedded probes for selective surface-enhanced Raman spectroscopy (SERS). The AuNR@MOFs are able to both take-up or block molecules from the pores, thereby facilitating highly-selective sensing at the AuNR ends. This proofof-principle study serves both to present the outstanding level of control in the synthesis as well as the high potential for AuNR@ZrMOF composites for SERS.
\end{abstract}

\section{Introduction}

Metal-organic frameworks (MOFs) are a class of crystalline materials constructed from inorganic metal clusters connected by organic linkers. ${ }^{1,2}$ They form three-dimensional open networks, placing them amongst the most porous materials reported, with surface areas as high as $8,000 \mathrm{~m}^{2} / \mathrm{g} .{ }^{1-5}$ With more than 84,000 structures reported so far at the Cambridge Structural Database, ${ }^{6}$ judicious choice of MOF construction by rational selection of the inorganic clusters and organic linkers provides a wide synthetic scope and allows for fine tuning of the textural properties and surface chemistry of the porous network. This makes MOFs a unique platform for gas adsorption and separation, ${ }^{7-11}$ heterogeneous catalysis, ${ }^{12}$ water treatment, ${ }^{13}$ sensing, ${ }^{14}$ and tumour diagnosis and drug delivery. ${ }^{15-17}$

Amongst many classes of MOFs, Zr-based MOFs featuring the hexazirconium oxocluster $\left(\left[\mathrm{Zr}_{6}\left(\mu_{3}-\mathrm{O}\right)_{4}\left(\mu_{3}-\mathrm{OH}\right)_{4}\right]^{12+}\right.$ or $\mathrm{Zr}$-cluster $)$, such as the pivotal UiO-66, have been of particular interest due to their exceptionally high thermal, mechanical and chemical stability. ${ }^{18,19}$ These MOFs are commonly synthesized solvothermally from $\mathrm{Zr}(\mathrm{IV})$-salts and polyvalent carboxylic acids. Notionally, one-pot syntheses can be separated into two steps: i) formation of Zr-clusters as rigid inorganic building blocks, and ii) framework self-assembly by connection of Zr-clusters via carboxylic acid linkers, through the COO-Zr binding motif. ${ }^{19} \mathrm{Zr}$ MOFs are often synthesized in the presence of a so-called modulator, a monovalent organic acid that competes with the polyvalent linkers for coordination to the Zr-clusters. ${ }^{19,20}$ Modulators improve the crystallinity of the MOFs and inhibit nucleation, thereby providing a powerful tool to control the size of the MOF-crystallites in the nano-range, i.e. nMOFs. ${ }^{21}$

The wide functional scope of pristine MOFs may be further expanded by inclusion of nanoparticle (NP) guests into the host framework, forming hybrid materials serving as platforms for catalysis, drug-delivery and sensing. ${ }^{22-26}$ Nanoparticle-MOF composites (NP@MOF) are commonly synthesized by bottlearound-ship methods, in which the MOF is assembled around the NPs. This kind of assembly can be classified based on growth mechanism and subsequent particle morphologies. If the NPs attach to the external surface of a pre-existing self-nucleating MOF crystallite, they will be immobilized inside the structure as consecutive layers of MOF grow around them. Such 'encapsulation' composites feature multiple NPs in the crystallite; spatial control and NP loading density can only be controlled by carefully timed addition of NPs to the MOF growth solution. ${ }^{23}$ On the other hand, if the NPs initiate the MOF crystallization as seeds, single NP ('core-shell') NP@MOF composites form. We note that 
such 'true' core-shell particles are more difficult to synthesize as they require precise matching of NP and MOF precursor concentrations as well as carefully tuned reaction kinetics to ensure that the MOFs do not self-nucleate. ${ }^{27,28}$ Although being easy to envision, a major difficulty in bottle-around-ship NPencapsulations is the thermal and salt-induced aggregation of NPs during the MOF growth, which severely limits the optimal production of NP@MOF composites. ${ }^{29}$ This is particularly true for Zr-based MOFs, which are commonly synthesized at high temperatures and for which only few core-shell nanostructures have been reported. ${ }^{30-32}$ In this regard, although recent advances in room-temperature syntheses of Zr-based MOFs by Farha and co-workers have started to improve the colloidal stability of NPs in bottle-around-ship assemblies, ${ }^{29,33}$ the field remains largely unexplored.

Looking at the applications of these composites, core-shell nMOFs provide a unique opportunity to harness the optical properties of noble metal NPs such as AuNRs for embedded spectroscopy probes. AuNRs are a common tool for surface enhanced Raman spectroscopy (SERS), which enhances the characteristic molecular Raman signal by multiple orders of magnitude.34-36 NP@MOF composites have been previously proposed as SERS probes due to their ability to selectively filter molecules as well as build up local concentrations of the analyte. ${ }^{37-41}$ However, these systems so far lack precise morphological control and it is unclear whether the NPs are fully encapsulated - required for filtering - or attached to the external surface of the crystallites. While the synthesis and SERS capability of a core-shell AuNR@ZIF-8 composite was demonstrated by Zheng et $\mathrm{al}^{\mathrm{4}}{ }^{2}$, the use of much more chemically stable Zr-MOFs for encapsulation remains unexplored till now due to the difficulty in their preparation. In addition, despite its flexibility, ${ }^{43}$ the use of a ZIF-8 shell limits the number of analytes that can access the porosity due to the very narrow $3.4 \AA$ window - a problem that can be overcome using other structures with larger pore sizes.

In this work, we present the room-temperature framework assembly of a novel AuNR@Zr-MOF core-shell composite. The encapsulating MOF used is the microporous NU-901 with scu topology which has a recently developed facile room-temperature synthesis. ${ }^{29} \mathrm{NU}-901$ has a relatively large aperture size capable of targeting large analytes. Scheme 1 shows the NU-901 structure and its constituents; similar to its well-known sister structure NU1000 with csq topology, NU-901 is comprised of eight-connected $\mathrm{Zr}_{6}\left(\mu_{3}-\mathrm{O}\right)_{4}\left(\mu_{3}-\mathrm{OH}\right)_{4}\left(\mathrm{H}_{2} \mathrm{O}\right)_{4}(\mathrm{OH})_{4}$ clusters which are linked by tetratopic 1,3,5,8-(p-benzoate)pyrene linkers ( $\mathrm{H}_{4}$ TBAPy). ${ }^{44-46}$ In both structures, the $\mathrm{Zr}$-clusters have eight loosely $\mathrm{H}_{2} \mathrm{O} / \mathrm{OH}$ coordinated sites as possible catalytic centers. The diamondshaped channels of NU-901 have a dimensions of ca. $12 \times 26 \AA$, which enables fast diffusion of relatively large analytes through the MOF. ${ }^{29}$ Embedding AuNRs inside as SERS probes allowed us to show size-selective uptake of analytes through the composite, and demonstrates for the first time selective uptake from a mixedanalyte solution.

\section{Results and Discussion}

We synthesized the AuNRs using the seed-mediated growth method developed by Nikoobakht et al. ${ }^{47,48}$ In our case, in order to stabilize the AuNRs in the MOF growth solution, we replaced the cetrimonium bromide (CTAB) capping ligand by thiolated polyethylene glycol (PEG-SH, Mw $=2,000 \mathrm{~g} / \mathrm{mol}$ ) using a phasetransfer method. ${ }^{49}$ We expected the PEG to interact with the MOF precursors due to its hard oxygen and soft ethylene moieties. UV/Vis-spectroscopy confirmed the successful dispersion in DMF, showing only small changes in the longitudinal surface plasmon resonance (LSPR) absorbance of the AuNR, consistent with the small change in optical dielectric constant at the particle-medium interface (Figure S1). a.

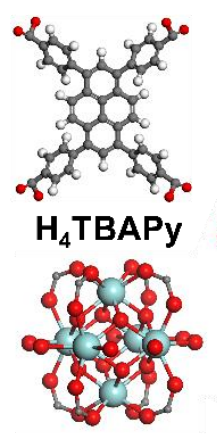

Zr-cluster

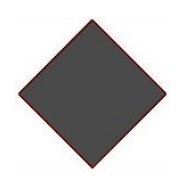

$\mathrm{H}_{4}$ TBAPy
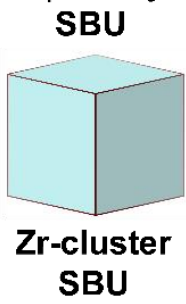

b.

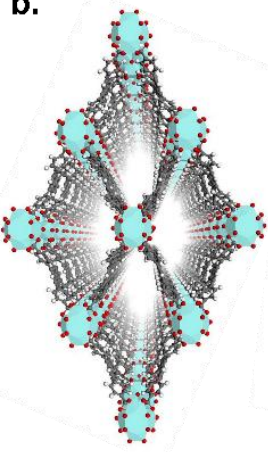

NU-901

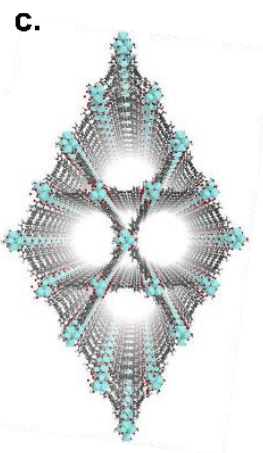

NU-1000
Scheme 1. a. MOF constituents $\mathrm{H}_{4}$ TBAPy linker and $\operatorname{Zr}_{6}\left(\mu_{3}-\mathrm{O}\right)_{4}\left(\mu_{3}-\right.$ $\mathrm{OH})_{4}\left(\mathrm{H}_{2} \mathrm{O}\right)_{4}(\mathrm{OH})_{4}$ cluster and corresponding SBUs. Carboxylate anchoring points drawn into $\mathrm{Zr}$-cluster, $\mathrm{H}$ atoms omitted for clarity b. Zr-MOF NU901, with scu topology. Pore dimensions: $12 \times 26 \AA$ c. Zr-MOF NU-1000 with csq topology. Pore dimensions: $12 \AA$ and $32 \AA$ for triangular pore and hexagonal pore, respectively.

After obtaining the AuNRs, we synthesized the core-shell crystallites using a two-step procedure adapted from Noh et al. ${ }^{29}$ Here, we first formed the Zr-clusters at high temperature, isolating them after. Second, we dispersed the isolated clusters in a mixture of DMF and acetic acid. The PEG-stabilized AuNRs (AuNR@PEG$\mathrm{SH})$ were added to this suspension, resulting in a deep-red 7:4 (v:v) mixture of DMF and acetic acid. Under vigorous stirring, we added an $\mathrm{H}_{4}$ TBAPy-linker solution in DMF, stirring the solution overnight. The dark-red precipitate was removed by centrifugation, washed extensively using DMF, and solvent-exchanged to acetone. Initial attempts to create core-shell structures using polyvinylpyrrolidone (PVP) capped AuNRs, a more conventional NP@MOF encapsulation agent, ${ }^{23}$ were unsuccessful and resulted in AuNR aggregation outside the NU-901 crystallites (Figure S2). Similarly, AuNRs functionalized with mercaptoundecanoic acid (MUA) aggregated during the synthesis and were not encapsulated by the MOF (Figure S2).

Figure 1 shows the transmission electron micrographs (TEM), powder X-ray diffraction (PXRD) patterns and $\mathrm{N}_{2}$ adsorption isotherms of the composites. The TEM images confirm the coreshell morphology of single AuNRs encapsulated by prolate NU-901 crystallites, hereafter denoted AuNR@NU-901. The morphology 
of the AuNR@NU-901 crystallites reproduces the reported prolate shape for pristine NU-901 particles, arising from the facet selectivities during the growth. ${ }^{21,29}$ Nearly all AuNRs are in the center of the crystallite and have a parallel orientation with a.

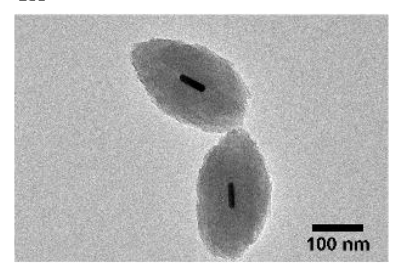

c.

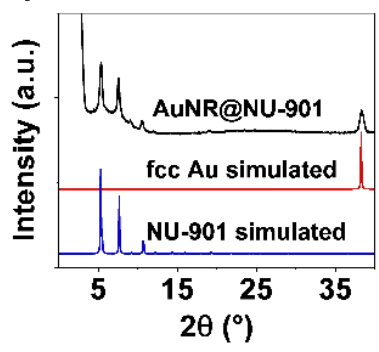

b.

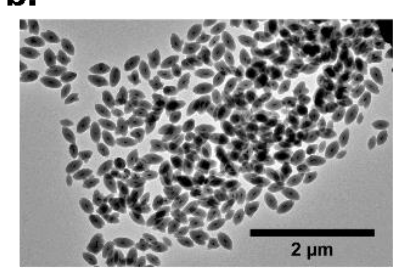

d.

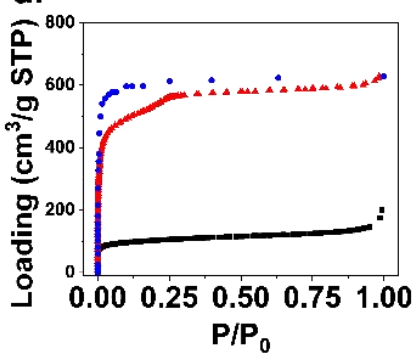

Figure 1. a, b. TEM micrographs of AuNR@NU-901. Note the parallel alignment of AuNR and principal axis of prolate crystallites c. Powder X-ray diffraction pattern of AuNR@NU-901. d. $N_{2}$ adsorption/desorption isotherm at $77 \mathrm{~K}$ of AuNR@NU-901 (black squares), pristine room-temperature-assembled NU-901 (RT NU-901, red triangles), simulated $\mathrm{N}_{2}$ adsorption isotherm on defect-free NU-901 (blue circles).

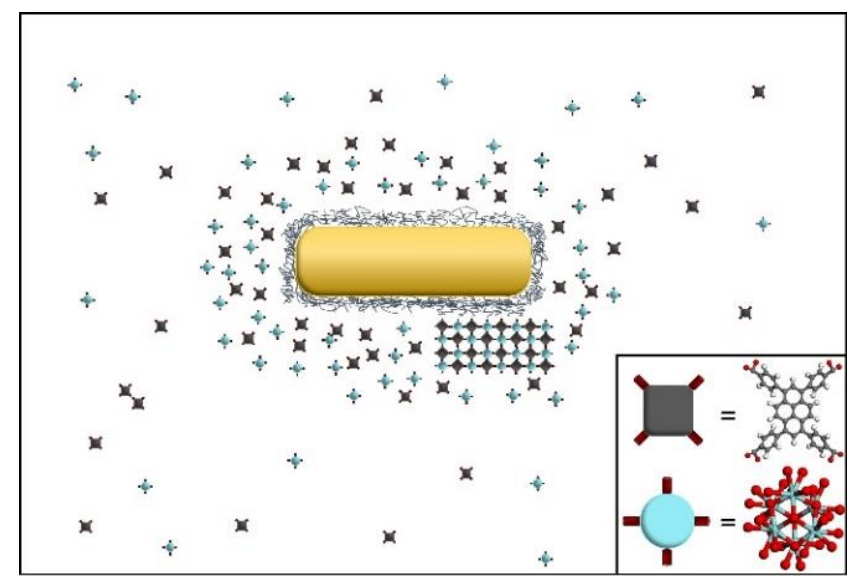

Scheme 2. Interactions between PEG and MOF precursors make nucleation more favorable on the AuNR than in free solution.

respect to the principal prolate axis. We also note that pristine NU-901 nanoparticles are larger than their core-shell counterparts and have a wider size-distribution (Figure S3). This suggests that the AuNR@PEG-SH seeds the NU-901 growth, presumably due to the interactions (dispersion forces, dipole-dipole interactions, etc.) between PEG and the MOF precursors. These interactions may raise the local concentration of the precursors on the surface of the AuNRs, making a nucleation event kinetically more favorable (Scheme 2). As the number of seeds is fixed by the concentration of AuNRs, a narrower size distribution results compared to pristine NU-901 (Figure S3).

The PXRD patterns of AuNR@NU-901 feature both low-angle diffractions from NU-901 $\left(2 \theta=5.3^{\circ}, 7.7^{\circ}, 10.7^{\circ}\right)$ and the (111)diffraction from fcc $\mathrm{Au}\left(2 \theta=38.2^{\circ}\right)$, confirming the crystallinity of the material (Figure 1c). The UV/Vis-spectrum of the composites contains bands corresponding to both the MOF ( $\mathrm{H}_{4}$ TBAPy) and AuNR absorbance (Figure S4). The Type I $\mathrm{N}_{2}$ adsorption isotherm at $77 \mathrm{~K}$ demonstrates that AuNR@NU-901 is microporous, albeit with a substantial decrease in the gravimetric porosity compared to the pristine NU-901 (Figure 1d). The calculated Brunauer-Emmett-Teller (BET) area of AuNR@NU-901 is 350 $\mathrm{m}^{2} / \mathrm{g}$, whilst that of pristine NU-901 is $2,000 \mathrm{~m}^{2} / \mathrm{g}$. We estimated the theoretical BET area by comparing the relative sizes of the particles and densities to calculate the mass contribution of NU901 (see Electronic Supplementary Information, ESI, for more details). For large AuNR@NU-901 crystallites (ca. $320 \mathrm{~nm}$ ) the theoretical BET area is roughly $1,800 \mathrm{~m}^{2} / \mathrm{g}$. We attribute the large reduction in gravimetric porosity either to a higher non-porous defect density in the smaller AuNR@NU-901 crystallites, such as collapsed pores and amorphous, non-porous phases, or to partial pore-blocking by the PEG surface ligands. Although NU-901 is an entirely microporous material, the Type IV $\mathrm{N}_{2}$ adsorption isotherm of the pristine room-temperature-assembled NU-901 (RT NU-901) features a characteristic 'mesoporous step' at 0.2 relative pressure, which Noh et al. attributed to porous crystal defects such as missing linkers and nodes. ${ }^{29}$ Indeed, a grand canonical Monte Carlo (GCMC) simulated adsorption isotherm on defectfree NU-901 shows an expected, typically microporous Type I isotherm. Interestingly, the $\mathrm{N}_{2}$ adsorption isotherm of AuNR@NU901 does not have a step, suggesting the removal of such mesoporous defects in our seeded growth. The shape of this isotherm is in better agreement with the GCMC simulated $\mathrm{N}_{2}$ adsorption isotherm (Figures $1 \mathrm{~d}$ and $\mathrm{S} 5$ ).

To further explore the synthesis of AuNR@NU-901, we changed the concentration of $\mathrm{H}_{4}$ TBAPy linker in the synthesis and monitored the results using TEM image analysis. Figure $2 \mathrm{a}$ shows a LaMer-type growth model we use here to discuss our results; note that this is a qualitative representation and does not show experimental results. First, the $\mathrm{H}_{4} \mathrm{TBAPy}$ concentration increases from zero, as the reagent is added to the Zr-cluster and AuNRbearing mother solution. Above the nucleation threshold, nucleation occurs followed by a simple first-order decay until the precursors are consumed or their concentration saturates. The model has two additional features in the nucleation and growth phase respectively: i) favorable interactions between the PEGylated AuNRs and the MOF precursors, which lower the nucleation threshold locally on the AuNRs, and ii) at a critical low concentration of $\mathrm{H}_{4}$ TBAPy during the growth phase, the topology changes from scu to csq, resulting in the less dense NU-1000 structure. ${ }^{45}$ We dub the difference between the MOF nucleation thresholds in solution ([Nuc solution]) and on the AuNRs ([NuC AunR]) the 'core-shell window'. Provided that the $\mathrm{H}_{4}$ TBAPy peakconcentration is within the core-shell window, nucleation will occur on the nanorods and core-shell crystallites are formed. Above the core-shell window, the concentration of MOF precursors is too high to discriminate effectively between the local concentrations on the AuNRs and in solution, forming nonencapsulated crystallites.

We estimated the core-shell yield (i.e. the fraction of crystallites with core-shell morphology) and size-distributions of different batches synthesized from varying $\mathrm{H}_{4}$ TBAPy concentrations using statistical analyses of TEM micrographs. Figure $2 b$ shows the analysis based on more than 150 particles. It shows that the coreshell yield decreases as the initial $\mathrm{H}_{4}$ TBAPy concentration increases, suggesting that the $\mathrm{H}_{4}$ TBAPy peak-concentration moves away from the core-shell window. Further, we observe that the 
average particle size becomes smaller as the core-shell yield decreases, owing to the greater number of seeds. Notably, nonencapsulated crystallites are consistently larger than their encapsulated counterparts. This fits our qualitative growth model, as it suggests that a higher fraction of non-encapsulated crystallites form at an earlier stage of the reaction, before the $\mathrm{H}_{4}$ TBAPy concentration has depleted to the core-shell window.

Interestingly, we observed a change in topology from scu (NU-901) to csq (NU-1000) during the growth phase at a critically low $\mathrm{H}_{4}$ TBAPy concentration. It has been previously shown that NU-1000 nMOFs are cylindrical as opposed to the prolate NU-901 nMOFs. ${ }^{15,21,29,44,50-52}$ Careful analysis of TEM micrographs shows that AuNR@NU-901 crystallites synthesized from low $\mathrm{H}_{4}$ TBAPy concentrations frequently have cylindrical rather than prolate ends. Under such fast-addition conditions, the nMOF morphology changes completely from prolate to cylindrical, if even lower concentrations are used (Figure 2c). These cylindrical nMOFs are best described as encapsulation composites rather than true coreshell, as they frequently contain multiple AuNRs randomly dispersed through the nMOFs. Direct measurement of the crystal lattice using high-resolution TEM (HRTEM) revealed a d-spacing of ca. $3 \mathrm{~nm}$ that is consistent with the NU-1000 phase (Figures S6 and
S7). To the best of our knowledge, this is the first reported evidence of NU-1000 formed in a room-temperature assembly. It has long been known that linker/modulator ratios affect the topology of Zr-MOFs; however a clear understanding is still lacking. ${ }^{52}$ We propose here that, as the $\mathrm{H}_{4}$ TBAPy is consumed in the reaction, the $\mathrm{H}_{4} \mathrm{TBAPY} / \mathrm{AcOH}$ ratio reaches a critically low value, at which point the NU-1000 phase is favored (Figure 2a, blue shaded area). Either exceeding the core-shell window, or growing mixed NU-901 and NU-1000 phases, can be avoided if the linker addition profile is modulated. Controlled dropwise injection (see the Electronic Supplementary Information for more details) using a syringe-pump increases the $\mathrm{H}_{4}$ TBAPy concentration more slowly (Figure 2a, dotted blue curve), ensuring that nucleation occurs only in the desired core-shell window (Figure 2a,b,f). Simultaneously, the linker concentration decreases more slowly compared to the fast injection method, since the reagent is continuously added, thereby preventing NU-1000 growth. Using this slow-injection method, we achieved core-shell yields greater than $99 \%$ as well as exclusively NU-901 crystallites. Interestingly, we did not observe a topological change from NU-901 to NU-1000 at low $\mathrm{H}_{4}$ TBAPy concentrations under slow-addition conditions (Figure S8). a.

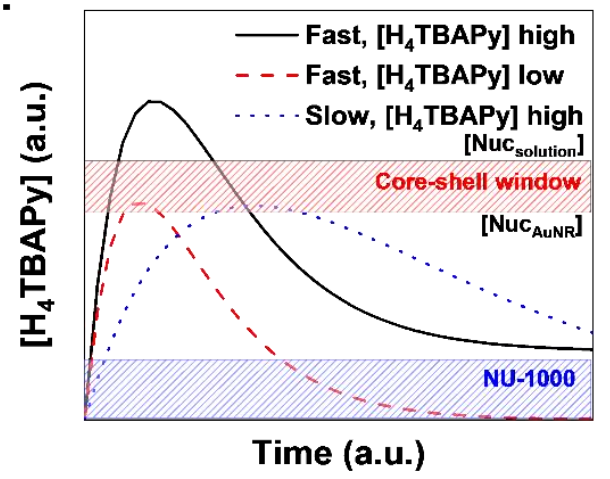

b.

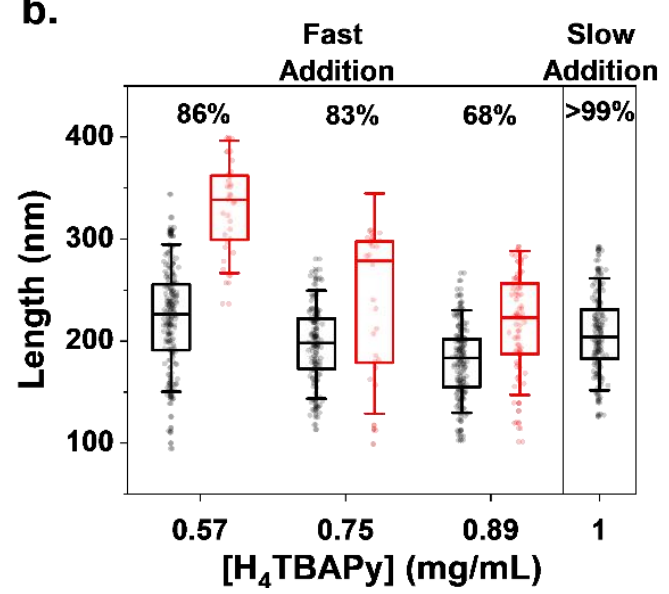

c.

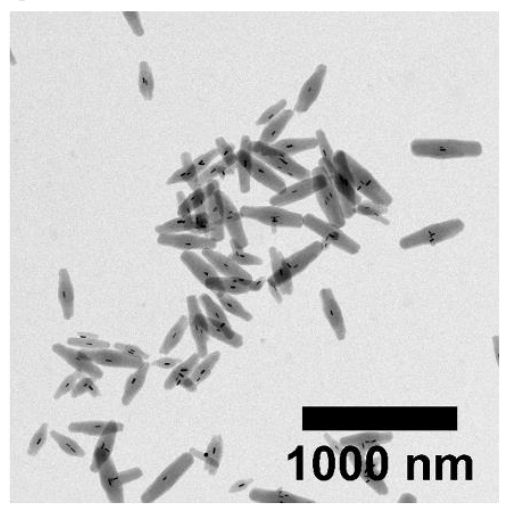

e.

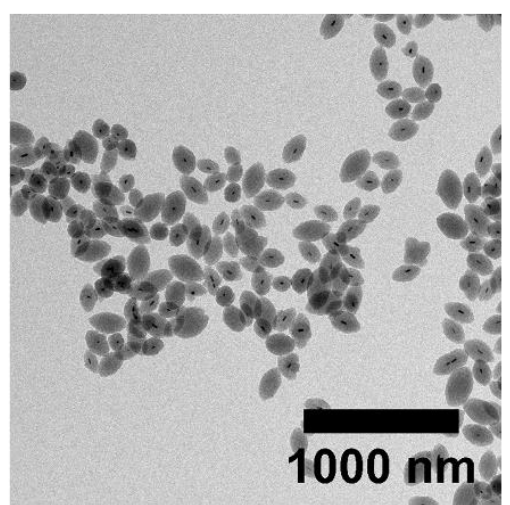

d.

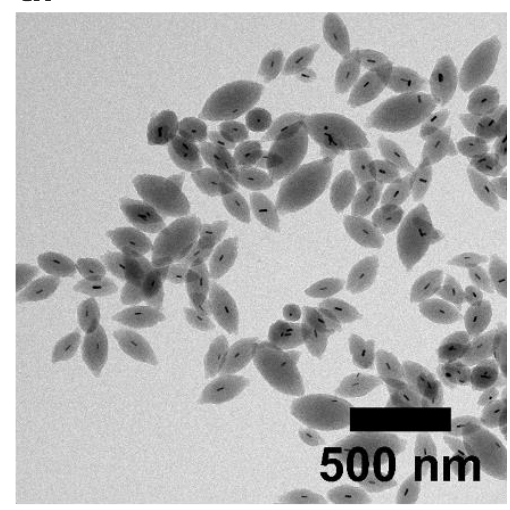

f.

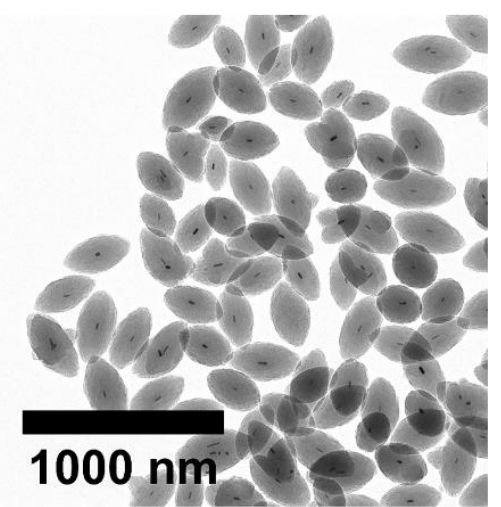

Figure 2. a. AuNR@NU-901 qualitative growth mechanism. Fast addition: $\mathrm{H}_{4}$ TBAPy solution added using pipette, slow addition: $\mathrm{H}_{4}$ TBAPy added dropwise using a syringe pump ( $0.005 \mathrm{~mL} / \mathrm{min})$ b. Statistical analysis of TEM micrographs of AuNR@NU-901 batches synthesized with different amounts of $\mathrm{H}_{4}$ TBAPy added (> 150 particles each). Length measured along the principal prolate axis. Top label gives core-shell yields; black points: core-shell crystallites; red points: non-encapsulated crystallites. Box position: average; line: median; box: interquartile range; whiskers: standard deviation c.-f. TEM micrographs of batches synthesized with different concentrations of $\mathrm{H}_{4}$ TBAPy: c. $0.33 \mathrm{mg} / \mathrm{mL}$; d. $0.57 \mathrm{mg} / \mathrm{mL} ; \mathbf{e} .0 .89 \mathrm{mg} / \mathrm{mL}$; f. $1.00 \mathrm{mg} / \mathrm{mL}$, slow addition (0.005 mL/min) 
At this point, we further control the size of the AuNR@NU-901 nMOFs by varying the concentration of AuNR seeds in the MOF mother solution. Assuming that the reaction rate is unaffected by the concentration of AuNRs, the particle size should decrease if the number of seeds is increased, as more particles will grow into smaller crystallites. Statistical analyses of TEM micrographs ( $>150$ particles each) were used to evaluate the size of the AuNR@NU901 composites. Figure 3 shows the relationship between particle size and AuNR concentration and their inverse relationship, as well as exemplary TEM micrographs. Batches containing higher concentrations of AuNRs yield smaller crystallites, while those containing fewer AuNRs result in larger particles. This intuitive result can be used to robustly tune the particle size between 200$350 \mathrm{~nm}$. Additionally, we have found that under slow-addition conditions, no distinct morphological changes have been observed and that hence the AuNR seed concentration is the dominant factor influencing the particle size (Figure S8).

High yields of fully encapsulated AuNR@NU-901 give a promising opportunity to exploit the SERS capabilities of the AuNRs as embedded probes for selective detection of molecular species diffusing inside the nMOFs. For SERS, the core-shell morphology is of particular interest, since it prevents SERS signal generation from non-encapsulated plasmonic material. The greatest extent of molecular filtration can thus be expected from core-shell particles and consequently the highest selectivity. To gain further insight into if the analyte fits inside the channels, we first used molecular dynamics to simulate diffusion of a single 4' mercapto-biphenylcarbonitrile (BPTCN) molecule in NU-901. BTPCN measures roughly $15 \times 7 \AA$ along the thiol-CN axis and phenyl axis respectively and should thus be small enough to traverse through the NU-901 channels. Figure S9 shows the density map of the trajectory of one molecule of BPTCN after a period $5 \mathrm{~ns}$ at room-temperature. This clearly shows that BPTCN can easily traverse not only the main channels but also from channel to channel through a hopping mechanism, confirming its ability to travel and diffuse through the channels to access the encapsulated AuNR. The videos provided in the ESI shows close proximity between BPTCN and the porphyrin rings suggesting $\pi-\pi$ stacking interactions.

a.

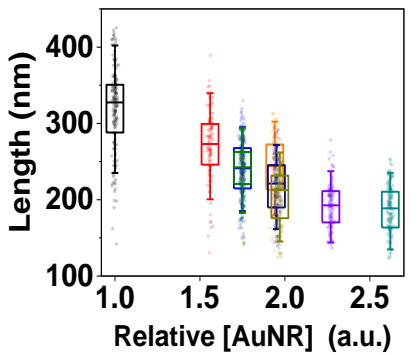

C.

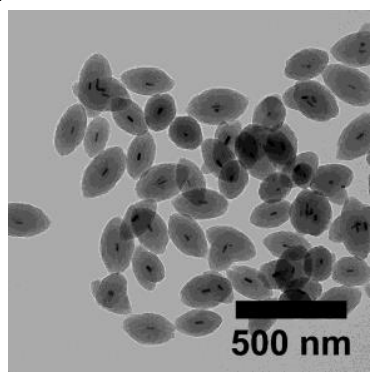

b.

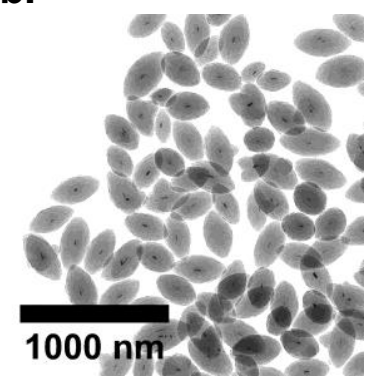

d.

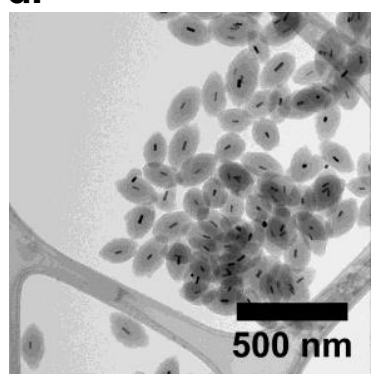

Figure 3. a. Statistical analysis of TEM micrographs of AuNR@NU-901 batches synthesized with different AuNR concentrations ( $>150$ particles each). Length measured along the principal axis of prolates. Box position: average; line: median; box: interquartile range; whiskers: standard deviation b.-d. TEM micrographs of AuNR@NU-901 batches synthesized with different AuNR concentrations. Relative concentrations: b. 1 c. 1.9 d. 2.6. Note that crystallite size decreases as the relative AuNR concentration increases.

Following the preliminary computational analysis, we incubated several batches of AuNR@MOFs with two aromatic thiolated analytes: BPTCN and biphenyl-thiol (BPT). We combined stock solutions of BPTCN and BPT with aliquots of AuNR@MOF suspensions in both acetone and $\mathrm{MeOH}$, and stirred overnight to allow diffusion into the porosity. After washing and drying the suspensions to remove excess of BPT/BPTCN from the external surface, we acquired the SERS spectra using a $785 \mathrm{~nm}$ laser in order to achieve efficient excitation into the plasmon bands (Figures S10, S4). The SERS spectrum of AuNR@NU-901 without the thiols is dominated by a large broadband emission (Figure S10). Control Raman spectra of its components in powder form (i.e. the isolated $\mathrm{Zr}$-cluster, $\mathrm{H}_{4} \mathrm{TBAPy}$, virgin NU-901, and AuNR@PEG-SH) show that AuNR@PEG-SH has a similar, unwanted, broadband emission (Figure S10). We thus subtracted polynomial fits to this AuNR@PEG-SH spectrum from all the MOF spectra. $\mathrm{H}_{4}$ TBAPy Raman modes are then clearly resolved in the AuNR@NU-901 spectra (Figure S10). We assigned the strongest modes at 1265 and $1609 \mathrm{~cm}^{-1}$ to ring breathing modes for the single and polyaromatic species, respectively. ${ }^{53}$ There is no evidence of Zr-cluster modes in the spectra, likely as their Raman cross-section is low relative to conjugated ring systems. ${ }^{54}$

Incubation of the core-shell nanocomposites in BPT results in a number of additional modes, particularly at 1078 and $1282 \mathrm{~cm}$ 1. These are assigned from density functional theory (DFT) calculations as $\delta(\mathrm{C}-\mathrm{H})$ and $v($ ring), respectively. Incubation in BPTCN also results in additional modes, at 1091, 1189, 1288 and $2230 \mathrm{~cm}^{-1}$, assigned from DFT as $\delta(\mathrm{C}-\mathrm{H})$ (thiol-phenyl ring), $\delta(\mathrm{C}-\mathrm{H})$ (both phenyl rings), $v($ ring), and $\mathrm{v}(\mathrm{C} \equiv \mathrm{N})$, respectively. Raman spectra from incubations of virgin NU-901 are inconclusive because there is no plasmonic enhancement effect. Very weak BPTCN modes are observed before washing and it is unclear if they are due to analyte inside or outside of the MOF. No BPTCN modes are observed after washing, which may be due to the removal of analyte from outside or inside of the crystals (Figure S11). These modes match well in position and intensity ratio to the spectra obtained through analyte incubation with AuNR@PEG-SH, indicating that the analytes have indeed infiltrated the NU-901 successfully to interact with the AuNR ends, where the plasmon field is concentrated. One notable exception is the intensity ratio of the nitrile mode at $2230 \mathrm{~cm}^{-1}$ to other BPTCN modes, which not as high in AuNR@NU-901 as in AuNR@PEG-SH (Figure S12).We suggest two different scenarios: i) the $v(C \equiv N)$ mode is preferentially enhanced at AuNR@PEG-SH compared to AuNR@NU-901, or ii) there are fewer nitrile groups in the AuNR@NU-901. The first scenario could occur due to orientation restraints imposed by the porosity, forcing nitrile groups to misalign with the field enhancement axis normal to the AuNR surface. This may occur if the molecule interacts with the AuNR at the nitrile rather than the thiol and is restrained from reorganizing. The second scenario could occur due to the hydrolysis or methanolysis of the nitrile group during diffusion, catalyzed by the Lewis-acidic Zr-cluster.

TEM analysis after the impregnation confirms that the coreshell structure was retained, and no free AuNRs observed (Figure 
S13). As a negative control, we incubated the AuNR@NU-901 with thiolated polystyrene (PST-SH; $\mathrm{Mw}=5,000 \mathrm{~g} / \mathrm{mol}$ ) to demonstrate the size selectivity of NU-901. Its Raman spectrum contains no distinct marker bands of PST-SH, which are however observed with isolated AuNR@PEG-SH (Figure 4a). We thus demonstrate the ability of NU-901 to block the entry of larger molecules into the pores, prohibiting diffusion to the AuNR core. For size selectivity to be a useful property, a MOF must be able to selectively filter species from a mixed solution. We incubated AuNR@NU-901 in a mixed solution of PST-SH and BPTCN, showing representative spectra in Figure 4 . This spectrum closely matches that of BPTCN incubated alone and shows no evidence of PST-SH adsorption.

a.
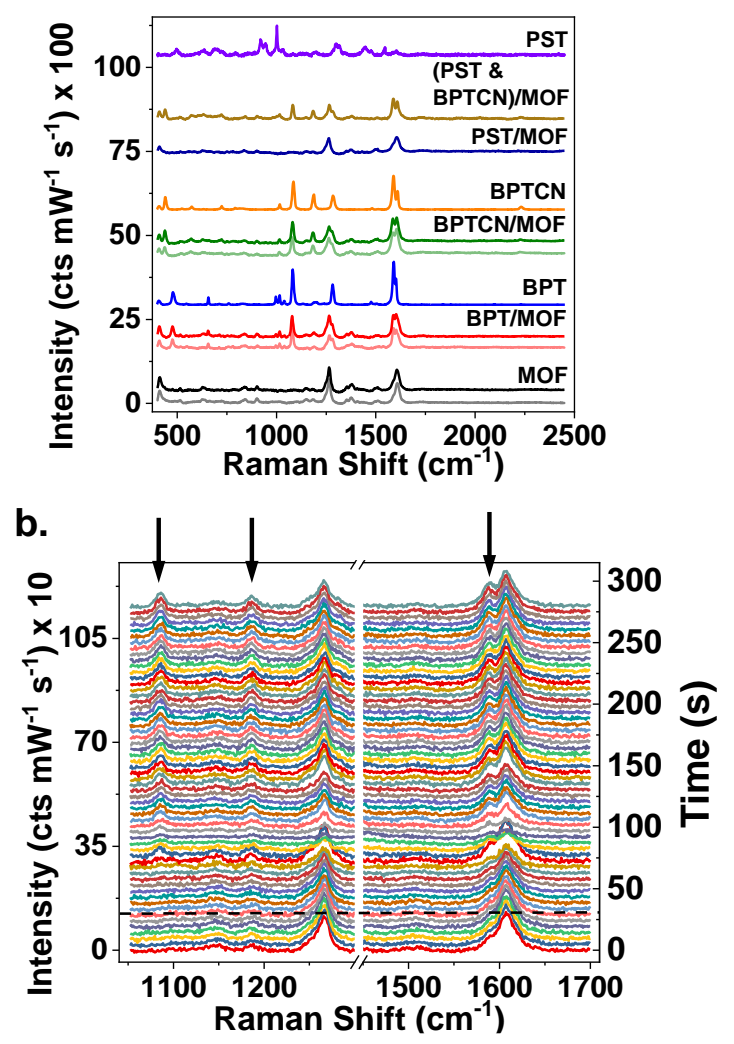

Figure 4. SERS spectra of a. AuNR@NU-901 (MOF) before and after incubation with various analytes (analyte/MOF). Spectra in lighter shades are acquired from incubation in $\mathrm{MeOH}$, all others in acetone. Spectra labelled only with analyte name are recorded from incubation with AuNR@PEG$\mathrm{SH}$, so exclude any spectral contributions or filtration effects from NU-901. All spectra are polynomial-fit background subtracted (see text) and scaled and offset for visual clarity. b. In-situ SERS measurements of BPTCN infiltration in an aqueous environment AuNR@NU-901. Spectra are background subtracted, dashed black line marks beginning of BPTCN addition. Arrows indicate analyte peaks.

To utilize the successful embedded probe technique further, we measured infiltration into the MOF in situ in an aqueous environment (Figure 4b). In experimental Raman studies, characteristic modes at 1091, 1189 and $1588 \mathrm{~cm}^{-1}$ appear in the spectrum immediately after injection of BPTCN, although those at 1189 and $1588 \mathrm{~cm}^{-1}$ overlap with weaker AuNR@NU-901 modes. The BPTCN mode intensity saturated $125 \mathrm{~s}$ after injection stops. Raman spectra of the solution away from AuNR@NU-901 (not shown) gave only weak scattering from the glass coverslip, confirming that the observed BPTCN modes do not originate from the surrounding solution. While slight focal drift occurs at the injection time, seen by a decrease in AuNR@NU-901 marker bands (1265 and $1608 \mathrm{~cm}^{-1}$ ), it is restored shortly after. To remove these focusing artefacts, the spectra are fitted to Lorentzian line shapes and integrated peak areas plotted as ratios of BPTCN to MOF peaks (Figure S14). These show clearly the expected first order increase in BPTCN:MOF SERS as soon as the BPTCN addition begins, directly tracking diffusion and binding of BPTCN inside the AuNR@NU-901 composite.

\section{Conclusion}

Here, we have demonstrated the tunable and versatile roomtemperature framework assembly of a novel AuNR@NU-901 coreshell composite, by using the AuNRs as seeds for the MOF growth. The core-shell yield is excellent (>99\%) if the $\mathrm{H}_{4}$ TBAPy-reagent is added dropwise using a syringe pump, and the final size of the composite can be controlled by changing the concentration of AuNR seeds in the mixture. The exploratory synthesis of AuNR@NU-901 also yielded first preliminary evidence for an NU1000 phase grown at room-temperature. From our in-depth exploration of the synthetic parameter space, we suggest that the pairing of specific NP surfactant and MOF is crucial to the encapsulation process. While this result is not a universal set of synthetic conditions to encapsulate metallic NPs by MOFs, we believe that we provide a valuable piece of evidence for other encapsulate systems in further work. We utilized the SERS capability of the AuNRs inside the MOF crystallites by infiltrating the MOFs with thiolated aromatic analytes. The resultant spectra clearly contain both MOF peaks and analyte peaks indicating successful adsorption. As a negative control, we incubated AuNR@NU-901 with a bulky polymer, which was subsequently blocked by the porosity of the MOF and not detected at the AuNR core. This proof-of-principle work sets the stage for both advanced MOF synthesis and embedded probe spectroscopy and provides a new capability for MOF plasmonics.

\section{ASSOCIATED CONTENT}

\section{Supporting Information}

The Supporting Information is available free of charge on the ACS Publications website.

\section{AUTHOR INFORMATION}

\section{Corresponding Authors}

*jjb12@cam.ac.uk

*farha-ofc@northwestern.edu

*df334@cam.ac.uk.

\section{Author Contributions}

§. W. M. O. and D. W. contributed equally.

Notes

The authors declare no competing financial interests.

\section{ACKNOWLEDGMENT}

This project has received funding from the European Research Council (ERC) under the European Union's Horizon 2020 research and innovation programme (NanoMOFdeli), ERC-2016-COG 726380, and LINASS 320503. The authors acknowledge the financial support from EPSRC grants EP/G060649/1, EP/L027151/1, and NanoDTC EP/L015978/1. D.F.-J. thanks the Royal Society for funding through a University Research Fellowship. H.N. gratefully 
acknowledges support from the Ryan Fellowship program of the Northwestern University International Institute of Nanotechnology. C.-W. K. acknowledges support from the Postdoctoral Research Abroad Program (105-2917-I-564-046) sponsored by Ministry of Science and Technology (Taiwan). All authors gratefully acknowledge Sanggyu Chong for his development of the simulation code RASPA.

\section{REFERENCES}

(1) Yaghi, O. M.; O'Keeffe, M.; Ockwig, N. W.; Chae, H. K.; Eddaoudi, M.; Kim, J. Reticular Synthesis and the Design of New Materials. Nature 2003, 423 (6941), 705-714.

(2) Furukawa, H.; Cordova, K. E.; O'Keeffe, M.; Yaghi, O. M. The Chemistry and Applications of Metal-Organic Frameworks. Science 2013 341 (6149), 1230444-1230444.

(3) Yaghi, O. M. Reticular Chemistry - Construction, Properties, and Precision Reactions of Frameworks. J. Am. Chem. Soc. 2016, 138 (48), 15507-15509.

(4) Zhou, H. C.; Long, J. R.; Yaghi, O. M. Introduction to Metal-Organic Frameworks. Chemical Reviews 2012, 112 (2), 673-674

(5) Li, S.; Huo, F. Metal-organic Framework Composites: From Fundamentals to Applications. Nanoscale 2015, 7, 7482.

(6) Moghadam, P. Z.; Li, A.; Wiggin, S. B.; Tao, A.; Maloney, A. G. P.; Wood, P. A.; Ward, S. C.; Fairen-Jimenez, D. Development of a Cambridge Structural Database Subset: A Collection of Metal-Organic Frameworks for Past, Present, and Future. Chem. Mater. 2017, 29 (7), 2618-2625.

(7) Li, J. R.; Sculley, J.; Zhou, H. C. Metal-Organic Frameworks for Separations. Chem. Rev. 2012, 112, 869-932.

(8) Farha, O. K.; Yazaydin, A. Ö.; Eryazici, I.; Malliakas, C. D.; Hauser, B. G.; Kanatzidis, M. G.; Nguyen, S. T.; Snurr, R. Q.; Hupp, J. T. De Novo Synthesis of a Metal-Organic Framework Material Featuring Ultrahigh Surface Area and Gas Storage Capacities. Nat. Chem. 2010, 2 (11), 944948.

(9) Li, B.; Wen, H. M.; Zhou, W.; Chen, B. Porous Metal-Organic Frameworks for Gas Storage and Separation: What, How, and Why? J. Phys. Chem. Lett. 2014, 5 (20), 3468-3479.

(10) Tian, T.; Zeng, Z.; Vulpe, D.; Casco, M. E.; Divitini, G.; Midgley, P. A.; Silvestre-Albero, J.; Tan, J. C.; Moghadam, P. Z.; Fairen-Jimenez, D. A SolGel Monolithic Metal-Organic Framework with Enhanced Methane Uptake. Nat. Mater. 2018, 17 (2), 174-179.

(11) Moghadam, P. Z.; Islamoglu, T.; Goswami, S.; Exley, J.; Fantham, M.; Kaminski, C. F.; Snurr, R. Q.; Farha, O. K.; Fairen-Jimenez, D. ComputerAided Discovery of a Metal-Organic Framework with Superior Oxygen Uptake. Nat. Commun. 2018, 9 (1), 1-8.

(12) Corma, A.; García, H.; Llabrés i Xamena, F. X. Engineering Metal Organic Frameworks for Heterogeneous Catalysis. Chem. Rev. 2010, 110, 4606-4655.

(13) Wang, C.; Liu, X.; Demir, N. K.; Chen, J. P.; Li, K. Applications of Water Stable Metal-Organic Frameworks. Chem. Soc. Rev. 2016, 45 (18), 51075134.

(14) Kreno, L. E.; Leong, K.; Farha, O. K.; Allendorf, M.; Duyne, R. P. Van; Hupp, J. T. Metal-Organic Framework Materials as Chemical Sensors. Chem. Rev. 2012, 112, 1105-1125.

(15) Teplensky, M. H.; Fantham, M.; Li, P.; Wang, T. C.; Mehta, J. P.; Young, L. J.; Moghadam, P. Z.; Hupp, J. T.; Farha, O. K.; Kaminski, C. F.; Fairen-Jimenez, D. Temperature Treatment of Highly Porous ZirconiumContaining Metal-Organic Frameworks Extends Drug Delivery Release. J. Am. Chem. Soc. 2017, 139 (22), 7522-7532.

(16) Horcajada, P.; Chalati, T.; Serre, C.; Gillet, B.; Sebrie, C.; Baati, T.; Eubank, J. F.; Heurtaux, D.; Clayette, P.; Kreuz, C.; Chang, J.; Hwang, K. H.; Marsaud, V.; Bories, P.; Cynober, L.; Gil, S.; Férey, G.; Couvreur, P.; Gref, R. Porous Metal-Organic-Framework Nanoscale Carriers as a Potential Platform for Drug Delivery and Imaging. Nat. Mater. 2010, 9 (2), 172-178. (17) Cavka, J. H.; Jakobsen, S.; Olsbye, U.; Guillou, N.; Lamberti, C.; Bordiga, S.; Lillerud, K. P. A New Zirconium Inorganic Building Brick Forming Metal Organic Frameworks with Exceptional Stability. J. Am. Chem. Soc. 2008, 130 (42), 13850-13851.
(18) Bai, Y.; Dou, Y.; Xie, L. H.; Rutledge, W.; Li, J. R.; Zhou, H. C. Zr-Based Metal-organic Frameworks: Design, Synthesis, Structure, and Applications. Chem. Soc. Rev. 2016, 45 (8), 2327-2367.

(19) Schaate, A.; Roy, P.; Godt, A.; Lippke, J.; Waltz, F.; Wiebcke, M.; Behrens, P. Modulated Synthesis of Zr-Based Metal-Organic Frameworks: From Nano to Single Crystals. Chem. - A Eur. J. 2011, 17 (24), 6643-6651.

(20) Majewski, M. B.; Noh, H.; Islamoglu, T.; Farha, O. K. NanoMOFs: Little Crystallites for Substantial Applications. J. Mater. Chem. A 2018, 6 (17), 7338-7350.

(21) Lu, G.; Li, S.; Guo, Z.; Farha, O. K.; Hauser, B. G.; Qi, X.; Wang, Y.; Wang, X.; Han, S.; Liu, X.; DuChene, J. S.; Zhang, H.; Zhang, Q.; Chen,X.; Ma, J.; Loo, S. C. J.; Wei, W. D.; Yang, Y.; Hupp, J. T.; Huo, F. Imparting Functionality to a Metal-organic Framework Material by Controlled Nanoparticle Encapsulation. Nat. Chem. 2012, 4 (4), 310-316.

(22) Moon, H. R.; Lim, D. W.; Suh, M. P. Fabrication of Metal Nanoparticles in Metal-organic Frameworks. Chem. Soc. Rev. 2013, 42 (4), 1807-1824.

(23) Hu, P.; Morabito, J. V.; Tsung, C. K. Core-Shell Catalysts of Metal Nanoparticle Core and Metal-Organic Framework Shell. ACS Catal. 2014, 4 (12), 4409-4419.

(24) Liu, Y.; Tang, Z. Multifunctional Nanoparticle@MOF Core-Shell Nanostructures. Adv. Mater. 2013, 25 (40), 5819-5825.

(25) Noh, H.; Kung, C. W.; Islamoglu, T.; Peters, A. W.; Liao, Y.; Li, P.; Garibay, S. J.; Zhang, X.; Destefano, M. R.; Hupp, J. T.; Farha, O. K.Room Temperature Synthesis of an 8-Connected Zr-Based Metal-Organic Framework for Top-Down Nanoparticle Encapsulation. Chem. Mater. 2018, 30 (7), 2193-2197.

(26) Rungtaweevoranit, B.; Baek, J.; Araujo, J. R.; Archanjo, B. S.; Choi, K. M.; Yaghi, O. M.; Somorjai, G. A. Copper Nanocrystals Encapsulated in ZrBased Metal-Organic Frameworks for Highly Selective $\mathrm{CO} 2$ Hydrogenation to Methanol. Nano Lett. 2016, 16 (12), 7645-7649.

(27) Zhou, J.; Wang, P.; Wang, C.; Goh, Y. T.; Fang, Z.; Messersmith, P. B.; Duan, H. Versatile Core-Shell Nanoparticle@Metal-Organic Framework Nanohybrids: Exploiting Mussel-Inspired Polydopamine for Tailored Structural Integration. ACS Nano 2015, 9 (7), 6951-6960.

(28) Zhao, H.-X.; Zou, Q.; Sun, S.-K.; Yu, C.; Zhang, X.; Li, R.-J.; Fu, Y.-Y. Theranostic Metal-organic Framework Core-shell Composites for Magnetic Resonance Imaging and Drug Delivery. Chem. Sci. 2016, 7 (8), 5294-5301.

(29) DeStefano, M. R.; Islamoglu, T.; Garibay, S. J.; Hupp, J. T.; Farha, O. K. Room-Temperature Synthesis of UiO-66 and Thermal Modulation of Densities of Defect Sites. Chem. Mater. 2017, 29 (3), 1357-1361.

(30) Odom, T.; Schatz, G. Introduction to Plasmonics. Chem. Rev. 2011 111, 3667-3668.

(31) Mayer, K. M.; Hafner, J. H. Localized Surface Plasmon Resonance Sensors. Chem. Rev. 2011, 111 (6), 3828-3857.

(32) Schlücker, S. Surface-Enhanced Raman Spectroscopy: Concepts and Chemical Applications. Angew. Chemie - Int. Ed. 2014, 53 (19), 4756-4795. (33) Sugikawa, K.; Furukawa, Y.; Sada, K. SERS-Active Metal-Organic Frameworks Embedding Gold Nanorods. Chem. Mater. 2011, 23 (13), 3132-3134.

(34) Sugikawa, K.; Nagata, S.; Furukawa, Y.; Kokado, K.; Sada, K. Stable and Functional Gold Nanorod Composites with a Metal-Organic Framework Crystalline Shell. Chem. Mater. 2013, 25 (13), 2565-2570.

(35) Liu, Y.; He, L.; Liu, Y.; Liu, J.; Xiong, Y.; Zheng, J.; Tang, Z. Core-Shell Noble-Metal@Metal-Organic-Framework Nanoparticles with Highly Selective Sensing Property. Angew. Chemie - Int. Ed. 2013, 52 (13), 37413745.

(36) Hu, Y.; Liao, J.; Wang, D.; Li, G. Fabrication of Gold NanoparticleEmbedded Metal - Organic Framework for Highly Sensitive SurfaceEnhanced Raman Scattering Detection. ACS Anal. Chem. 2014, 86, 39553963.

(37) Cao, X.; Hong, S.; Jiang, Z.; She, Y.; Wang, S.; Zhang, C.; Li, H.; Jin, F.; Jin, M.; Wang, J. SERS-Active Metal-organic Frameworks with Embedded Gold Nanoparticles. Analyst 2017, 142 (14), 2640-2647.

(38) Zheng, G.; de Marchi, S.; López-Puente, V.; Sentosun, K.; Polavarapu, L.; Pérez-Juste, I.; Hill, E. H.; Bals, S.; Liz-Marzán, L. M.; Pastoriza-Santos, I.; Pérez-Juste, J. Encapsulation of Single Plasmonic Nanoparticles within ZIF-8 and SERS Analysis of the MOF Flexibility. Small 2016, 3935-3943. 
(39) Fairen-Jimenez, D.; Moggach, S. A.; Wharmby, M. T.; Wright, P. A.; Parsons, S.; Düren, T. Opening the Gate: Framework Flexibility in ZIF-8 Explored by Experiments and Simulations. J. Am. Chem. Soc. 2011, 133 (23), 8900-8902.

(40) Li, P.; Klet, R. C.; Moon, S.-Y.; Wang, T. C.; Deria, P.; Peters, A. W.; Klahr, B. M.; Park, H.-J.; Al-Juaid, S. S.; Hupp, J. T.; Farha, O. K. Synthesis of Nanocrystals of Zr-Based Metal-organic Frameworks with Csq-Net: Significant Enhancement in the Degradation of a Nerve Agent Simulant. Chem. Commun. 2015, 51 (54), 10925-10928.

(41) Mondloch, J. E.; Bury, W.; Fairen-Jimenez, D.; Kwon, S.; Demarco, E. J.; Weston, M. H.; Sarjeant, A. A.; Nguyen, S. T.; Stair, P. C.; Snurr, R. Q.; Farha, O. K.; Hupp, J. T. Vapor-Phase Metalation by Atomic Layer Deposition in a Metal-Organic Framework. J. Am. Chem. Soc. 2013, 135 (28), 10294-10297.

(42) Kung, C. W.; Wang, T. C.; Mondloch, J. E.; Fairen-Jimenez, D.; Gardner, D. M.; Bury, W.; Klingsporn, J. M.; Barnes, J. C.; Van Duyne, R.; Stoddart, J. F.; Wasielewski, M. R.; Farha, O. K.; Hupp, J. T. Metal-Organic Framework Thin Films Composed of Free-Standing Acicular Nanorods Exhibiting Reversible Electrochromism. Chem. Mater. 2013, 25 (24), 50125017.

(43) Nikoobakht, B.; El-Sayed, M. A. Preparation and Growth Mechanism of Gold Nanorods (NRs) Using Seed-Mediated Growth Method. Chem. Mater. 2003, 15 (10), 1957-1962.
(44) Scarabelli, L.; Sánchez-Iglesias, A.; Pérez-Juste, J.; Liz-Marzán, L. M. A "Tips and Tricks" Practical Guide to the Synthesis of Gold Nanorods. J. Phys. Chem. Lett. 2015, 6 (21), 4270-4279.

(45) Alkilany, A. M.; Yaseen, A. I. B.; Park, J.; Eller, J. R.; Murphy, C. J. Facile Phase Transfer of Gold Nanoparticles from Aqueous Solution to Organic Solvents with Thiolated Poly(Ethylene Glycol). RSC Adv. 2014, 4 (95), 52676-52679.

(46) Deria, P.; Yu, J.; Smith, T.; Balaraman, R. P. Ground-State versus Excited-State Interchromophoric Interaction: Topology Dependent Excimer Contribution in Metal-Organic Framework Photophysics. J. Am. Chem. Soc. 2017, 139 (16), 5973-5983.

(47) Webber, T. E.; Liu, W. G.; Desai, S. P.; Lu, C. C.; Truhlar, D. G.; Penn, R. L. Role of a Modulator in the Synthesis of Phase-Pure NU-1000. ACS Appl. Mater. Interfaces 2017, 9 (45), 39342-39346.

(48) Islamoglu, T.; Otake, K.; Li, P.; Buru, C. T.; Peters, A. W.; Akpinar, I.; Garibay, S. J.; Farha, O. K. Revisiting the Structural Homogeneity of NU1000, a Zr-Based metal-organic Framework. CrystEngComm 2018, 20, 5913-5918

(49) Chua, Y. T.; Stair, P. C. An Ultraviolet Raman Spectroscopic Study of Coke Formation in Methanol to Hydrocarbons Conversion over Zeolite $\mathrm{H}$ MFI. J. Catal. 2003, 213 (1), 39-46.

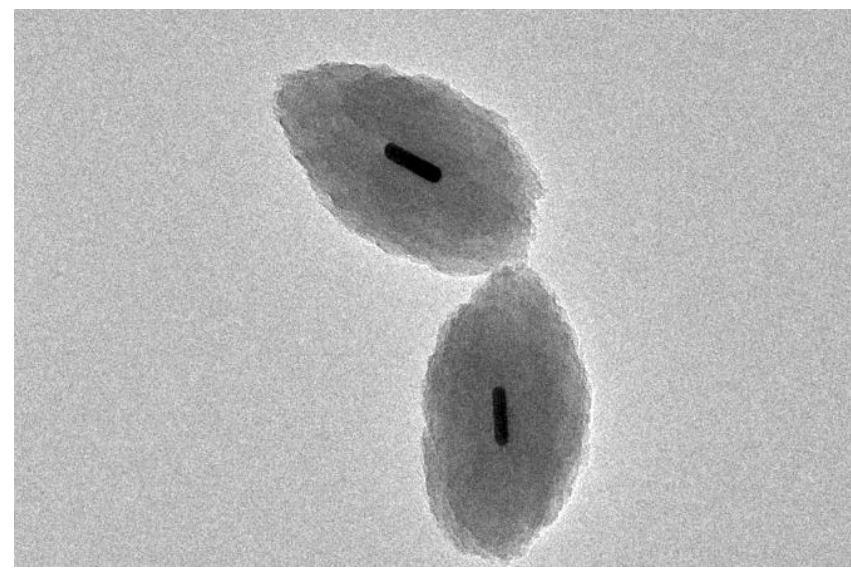

